\title{
A Literature Review on Optimal Placement of PMU and Voltage Stability
}

\author{
Dolly Chouhan and Varsha Jaiswal
}

School of Electronics and Electrical Engineering, Lovely Professional University, Punjab, India; dollychouhan18@gmail.com, jaiswal.varsha12@gmail.com

\begin{abstract}
In the present scenario, it is difficult to manipulate the nature of the power system which makes the system unstable. Therefore, real time monitoring is needed for checking out the possible outcomes of the failure in the system. Hence Phasor Measurement Unit (PMU) proves to be promising device as its gaining importance for its wide area application in real time which will be beneficial from both these aspect i.e reduction of contingencies and improving security system. This paper focuses on the various optimal placement techniques of PMU, discusses about the voltage stability and shows some of the real time applications of PMU.
\end{abstract}

Keywords: Global Positioning System (GPS), Phasor Measurement Unit (PMU), Observability Optimal Placement of PMU (OPP), State Estimation, Voltage Stability

\section{Introduction}

Being dynamic in nature, power system is prone to many contingencies and disturbance and in recent years, major blackouts have been recorded throughout the world. Disturbances for example, loss of line or generators, surges, faults, changing of load and so on can cause the system's operating points to deviate from the limits. In order to account on these problems and to find out the reasons of their occurring, one has to keep a check on parameters related to all these phenomenon i.e., voltage which will also incorporate the abnormal conditions like transient instability, oscillations and so on. Hence it is needed to maintain the steady limits of the voltage within the range i.e.; before and after the disturbance. Several researches have been done in corrective control for strategic static voltage stability. Studying and controlling voltage collapse occurrences will require dynamic and static techniques which will helps in effective planning of the system with greater reliable operation of interdependent critical infrastructures. Therefore real time monitoring of the system is required for the faster control providing online solutions and implementation so as to mitigate these challenging problems. Synchronized phasor measurement technology is relatively new, based on this technology, several application have been developed. This paper presents a literature review in the following sections as: Section I gives the need for the Phasor Measurement Unit (PMU), Section II has the review of PMU and its application, Section III and IV shows Optimal Placement of PMU (OPP) techniques, Section V and VI gives PMU based voltage stability and in last Section VII concludes the paper.

\section{Phasor Measurement Unit}

To represent steady state sinusoidal waveform of fundamental power frequency, phasors are used which are considered as basic tools for analysis of ac circuit. Moreover, it can be used to describe dynamic behavior of the power system where variations can be recognized as a series of steady state conditions. We can have efficient methods to deal with continuous monitoring of the input waveform known as recursive form of phasor equation by which we can measure all the three phases and can find out the positive sequence phasors.

${ }^{*}$ Author for correspondence 


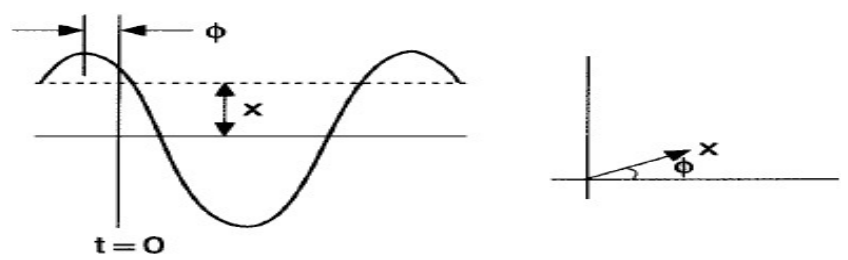

Figure 1. Phasor representation of sinusoidal waveform ${ }^{4}$

Here on a common reference base, voltages and currents are firstly measured and then converted to phasors. Later on precisely sampled with the help of satellite transmissions i.e.; Global Positioning System (GPS). Earlier the functionality of the GPS is meant for only navigation purposes. Synchronization is useful for distant substations which can be economical for error of less than 1microsecond.

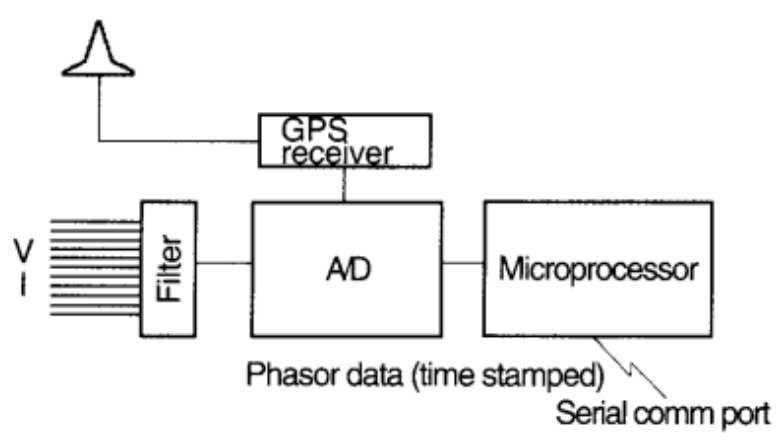

Figure 2. Major elements of $\mathrm{PMU}^{4}$

Main component of PMU are:

GPS receiver: It provides the 1 pulse-per-second (pps) signal, and a time tag, which contains the year, day, hour, minute, and second where time can be the local or the UTC (Universal Time Coordinated).

Phase-locked oscillator: It divides this 1 pps into the desired number of pulses per second which is needed for sampling of the analog signals. Generally it will be 12 times per cycle of the given fundamental frequency. Antialiasing and

Surge filtering: They provide the same phase shift and attenuation of output of the secondary of voltage and current transformer which assures that the differences of phase angle with their relative magnitudes are unchanged.

Microprocessor: It determines the positive sequence of phasor defined from recursive algorithm with the GPS timing message having sample number assigned to the phasor as its identifying tag at the beginning of the window. In an assembled message stream the computed string of phasors for each phases got communicated to a remote site through the modems ${ }^{1}$.

Though the fully utilization of the PMU technology has not been done. Some of their prototypes are being used in the system and have admirable performance in all respect as they proved to be the promising in their applied fields ${ }^{2-4}$.

a) Measurement of phasor's frequency and magnitude: As no need of synchronization of sampling signals as they are based upon the Fourier-Transform which provides high precision calculations. Use of recursive positive sequence calculation method is there. Complex number will rotate in a complex plane with frequency sampling clock fixed. Here the angular velocity is said to be equal to the differences of the nominal and the actual power system frequency.

b) State Estimation: By using the different measurement data received from different substations, applying an iterative nonlinear procedure, state of the power system can be done. This data is taken from the first measurement time when the state estimate is available to the several times it get elapsed. But the state estimators available is used to steady state only.

c) Instability Prediction: It improves the protection and control systems responses.For real-time stability analysis, transient energy function can be used.

d) Adaptive Relaying: By sensing the extreme values of the parameter changes like impedance and what time it will take to bring the change, the result can be inferred whether the power swing is stable or unstable. If the outcome can be deducted from the real-time data given by the synchronized phasor measurements earlier, it will gives us a idea for a fitting response that will be much more closer to the solution needed in existing power system $\frac{1,2,4}{\text {. }}$.

\section{Optimal Placement of PMU}

Since it is not economical to place PMUs at each and every bus to diagnose/detect the problematic area, so we need a placement technique which will provide a complete observability of the system. Placement techniques will depend on the PMUs application, installation restriction and the system characteristics as it is not even necessary also to place at each and every bus.

Many topological and numerical methods are there to determine the optimal location of PMUs. But we aims to 
find out the one technique or the combination of techniques which will not only minimize the installation cost but will provide full observability using a minimum set of phasor measurements.

Earlier to solve the OPP problem, Methods Like Linear (LP), non linear (NLP), dynamic programming or combinational optimization are used. These techniques are all conventional optimization techniques. But they have certain disadvantages like handling of constraints are complex, tedious, or numerical difficulties. Therefore other optimization techniques i.e.; heuristic and modern meta heuristic are there. These may include Minimum Spanning Tree (MST), Depth First Search (DeFS), Tabu Search (TS), Simulated Annealing (SA), Differential Evolution (DE), Genetic Algorithms (GA), Ant Colony Optimization (ACO). Immune Algorithms (IA), or Particle Swarm Optimization (PSO).

\section{Mathematical Programming Methods}

\subsection{Conventional Method}

\subsubsection{Integer Linear Programming (ILP)}

Here the design variables takes integer values.

$\mathrm{In}^{\underline{5}}$ objective is to the minimize the no. of located PMUs that will eliminate the measurement issue in the whole system. This is then further extended to incorporate conventional measurements. Again the same formulation is used for local redundancy. It deals with the different degrees of vulnerability with respect to measurements losses and bad data.

In ${ }^{6}$ aims for placement of PMUs properly according to a given budget. Here a special case of ILP called as Binary Integer Programming (BIP) is used. Here injection and measurement of power flow is considered. In addition to this, failure of single PMUs is accounted so that error related to state estimation to PMU failures can be minimized.

$\operatorname{In}^{\underline{7}}$ with and without zero injections situations are taken into the account. Modelling is done linearly and solved by ILP taking full and incomplete observability.

$\mathrm{In}^{-\frac{8}{}}$ partitions of the spanning tree of network is done using a two level approach which results into two or more sub networks with the help of ILP. Formulation of ILP is based on eigenvectors obtained from the spanning tree's adjacency matrix. In order to minimize the installation cost, after decomposition, place the PMUs optimally in the sub networks.

\subsubsection{Integer Quadratic Programming (IQP)}

It will optimize the quadratic objective function which has linear constrained and here also design variable takes integer value.

$\mathrm{In}^{9}$ process will minimize the number of PMUs maintaining complete observability under normal as well as under outage conditions. Hence it will give us a beneficial aspect of redundancy. Various IEEE test systems are used to check it taking into the account of the failure of single line or PMU.

In ${ }^{10}$ solution of OPP problem is deducted by using the connectivity matrix which represents network topology and then beneficial to formulate the optimization problem.

\subsubsection{Greedy Algorithm}

As it its name signifies, it takes the best immediate while finding an answer. It is a combinatorial optimization algorithm.

In ${ }^{11}$ to reduce the placement model size and it's the computational effort, a matrix reduction algorithm as well as virtual data elimination pre-processing method is used.

\subsection{Heuristic Methods}

\subsubsection{Minimum Spanning Tree (MST)}

Modified versions of depth first approach method. Proves to be advantageous over MST algorithm improves the DeFS approach as it has fast computing characteristics, reduces complexity and weak convergence of $\mathrm{DeFS}^{12}$. Use of this algorithm can be seen in China's Yunnan Power $\operatorname{grid}^{12}$.

\subsection{Metaheuristic Methods}

\subsubsection{Simulated Annealing (SA)}

It provides a good solution by trying random variations of the present solution. The algorithm will find an optimal or nearer to it solution if the cooling schedule gets slower or decreases its rate. 
$\mathrm{In}^{\frac{13}{3}}$ simple objective function is accounted where the installation cost and the distribution cost of the measuring devices is also considered.

In ${ }^{14}$ SSA method i.e.; stochastic simulated annealing is used. It is also used to verify and satisfy topological observability. Here PMUs are placed in such a way that they results in a measurement system but does not uses critical measurements. These will detect any single measurement containing bad data. As a penalty factor, inclusion of Critical measurement identification is there.

Likewise again a similar method is there in ${ }^{15}$. Here on the basis of volume of initial information which we get from Supervisory Control And Data Acquisition (SCADA) and PMU measurement can determine all the components of state vector required for load flow without the use of iteration which will minimize the required number of PMUs.

\subsubsection{Genetic Algorithm (GA)}

It is a type of search heuristic which involves the natural evolution process. Hence it is used to provide useful solutions for optimization and problem searches.

In ${ }^{16}$ different PMU placement situations are used to provide optimal solutions where criteria such as the critical measurements and sets from the system, maximum value of measured quantity obtained which are compared to the initial one, estimate's maximum accuracy, PMUs minimum cost, and transforming of the graph into tree are absent.

$\mathrm{In}^{17} \mathrm{GA}$ approach is used to the OPP problem. Accuracy of estimator is calculated by using fitness function for the GA .Results show that the use of PMUs will produce increase of the fitness function by 4 times.

$\operatorname{In}^{18}$ a distinctive aspect can be seen which provides a realism effect which is not there in others technique where the individuals are coded in the GA. This allows a fast and distinctive quantification of fitness value for each individual. Here optimal numbers of PMUs are used with minimal phasors.

\subsubsection{Tabu Search (TS)}

It is a combinatorial search technique. It is used for solving OPP with the help of using tracking and guiding. It will require less computation time and is highly robust. Hence making it faster than any conventional methods.
$\operatorname{In}^{19}$ author proposed a combination of augment incidence matrix with TS algorithm which is used for the OPP problems.

\subsubsection{Immune Algorithm (IA)}

It is based on genetic algorithm principles and here the mechanism of protection of living organisms from viruses and bacteria are there ${ }^{20}$.

\subsubsection{Particle Swarm Optimization (PSO)}

It provides a population-based search step where individuals are called particles whose position keeps on changes with respect to time. Here the particles flie in a multidimensional search space. When the particles fly, they adjust their own position according to their own and neighboring particle's experience. Hence makes the best use of positions. In these the set of particles and its previous history will determine the swarm direction.

$\mathrm{In}^{21}$ combination of the GA algorithm and PSO algorithm is used effectively. The characteristic of cross mutations of GA are used to decrease the scope of search of the PSO method. Hence will improve initial PMU placement quality which will improve or boost its solving process speed. A unique property i.e.; pseudo measurement is proposed which is nothing but a speedy observability analysis method.

$\mathrm{In}^{22}$ a modified BPSO algorithm is shown which will reduce the PMUs requirement and place them optimally with constraints satisfied.

$\mathrm{In}^{2} \underline{23}$ a combination of immune and BPSO algorithm is introduced. In this swiftness of BPSO with antibodies diversity provides the ability of convergence.

\subsubsection{Ant Colony Optimization (ACO $)^{24}$}

It's a classical probabilistic technique which uses graphs which will reduce computational problems complexity.

\section{PMU-Based Voltage Instability Monitoring}

Voltage stability can be defined as the ability of the system to maintain acceptable and steady voltage across all the buses even after being subjected to a disturbances. It is one of the category of the power system stability. 


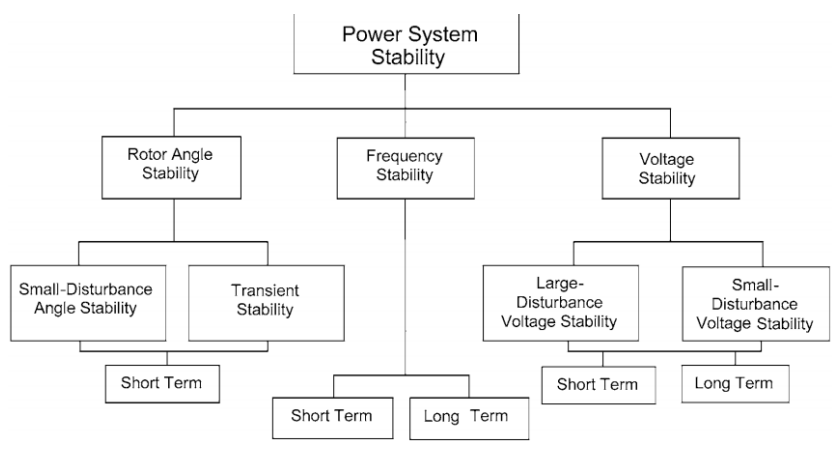

Figure 3. Classification of power system stability 25

Any one of these reasons can cause voltage instability:sudden increment of load demand, loss of load, tripping of lines and other devices by their protective system and so on resulting in progressive and uncontrollable voltage drop ${ }^{25}$. One of the major reasons is the insufficient fulfillment of reactive demand by the system devices. Even for at least one bus in the system if the voltage is unstable, the magnitude of bus voltage will get decreases as the injection of the reactive power in that bus will get increased. Voltage collapse can also be taken into glance which implies to the process where consecutive events happens accompanying voltage instability gives rise to either blackout or abnormal low voltages. Factors affecting the system due to collapse will be strength of transmission systems, characteristics of load, reactive power capability of generator limits and so on. Classification of voltage stability can be done with respect to time frame of their evolution (short term or long term stability) or with respect to the disturbance level (large or small) ${ }^{25}$. Some conventional methods exist for steady state voltage stability analysis such as P-V curve method, V-Q curve, Power-flow analysis and continuation power flow method. Since finding out the optimal solution will be difficult, assumptions are to be made. Several methods are categorized as Static, Dynamic and Quasi state Analysis which owns unique approach and property along with some merits and demerits ${ }^{25}$.

Two broad methodology based on local measurements and observability of the whole region. In the local measurement method few or no exchange of information between the locations are to be monitored and can be implemented in a distributed manner. The time skew of SCADA data is stored hence no need of time synchronization. Majorly they account for the Thevenin impedance matching or its extensions with the assumption that voltage instability is approximately similar to that of the maximum load capability of a network whereas in the observability of the whole region requires time-synchronized measurement where fitting of algebraic equations to the sampled states is done which are computed from the data of PMUs measurements and gives various sensitivity factors and the indices associated with them ${ }^{2}$.

In ${ }^{26}$ the authors showed optimal placement of PMU to get the data for state estimation, improves the reliability of the system, the voltage stability analysis using indicesFast Voltage Stability Index (FVSI) and Line Stability Index (LQP). Here the results of voltage and phase angle for IEEE-14 test system are then compared in two conditions i.e; with or without the PMU algorithms; Weighted Least Squares (WLS) and Least Absolute Value (LAV) along with their algorithms where LAV proved to be good and inclusion of PMU leads to the error reduction. For reducing the no. of PMU needed, Zero injection busses are considered.

$\mathrm{In}^{27}$ the authors presented the method how to calculate voltage stability margin when generator reactive power limits are taken for practical consideration. It makes the system to get a practical platform when reactive power violates their limit. Techniques used are coupled single-port Thevenin equivalent model and cubic spline extrapolation. They are used to find out the voltage collapse .Techniques algorithms and several other voltage stability margins are presented and discussed with the help of which we can find out the extreme reactive power violation. The aim is to know the problem caused by random load disturbances of data inconsistencies and their uncertainties. IEEE test systems are used to demonstrate the effectiveness of the proposed method.

In ${ }^{28}$ what the authors have tried to do that they have taken IEEE-30 bus system for checking out their simulation result. Here they have taken few buses with heavy loads on it and calculated that those few buses will be the having impact on the voltage stability of the system .For this they have chosen analytical index method both for online assessment of voltage stability and for optimal placement of PMU overcoming the cons of use of thevenin model method. Here it is shown how the impedance ratio will varies with the scaling factor and when it comes to or nearer to unity, a state of voltage collapse has been deducted with the increase in the scaling factor.

$\operatorname{In}^{\underline{29}}$ the author has described Echo State Network (ESN), a type of recurrent neural network. Here they have estimated Voltage Stability Load Index (VSLI) from optimally placed PMU which were found taken the islanding operating conditions into the consideration. This method 
is fast and has greater accuracy than any other methods. Test bus is IEEE- 14 bus system and simulated for both the normal and islanded system on the Real Time Digital Simulator (RTDS) system. Optimal placement for the PMU is found out by Genetic algorithm and described the need for optimal placement when the islanding is done as the system varies and hence the voltage stability. VSLI is also obtained from the thevenin network providing the fact that at no load its voltage value is zero while at the voltage collapse it will go to unity. ESN algorithm and method are also described.

$\mathrm{In}^{30}$ the authors presented completely observable system with the help of topological observability method so as to calculate the minimum no. of PMUs. Method whose placement are done using the conventional method and injection method full observability. Data collected from them are in real time current and voltage phasors which is for finding out the L-index needed to assess the voltage stability in between the points of normal as well as the instable operating point. Simulation is done 0n IEEE-14 bus and binary integer programming method is used.

$\mathrm{In}^{31}$ the authors have deducted the online load identification method needed to be studied as due the cascading tripping and blackout makes a state of voltage collapse will occur. On the basis of different type of loads characteristics, different protection system is used. Hence they have formulated the adaptive voltage control method along with the protection scheme like On-Load Tap Changer (OLTC) as the PMU alone can't mitigate these collapses if we concern for the long term. Hence it will not reduce the requirement of PMUs but also the load shedding problem. Here 4 bus test system is used.

$\mathrm{In}^{22}$ book, the author B.R Gupta has explained the load flow analysis and its techniques which is well applicable in finding out the optimal solution which can further be used to detect the voltage stability and its parametric indices. Moreover he gave the introduction to these conventional methods.

$\operatorname{In}^{33}$ the authors have commendably appreciated the applications of PMU and explained them thoroughly. They have highlighted every aspect of the synchronized phasor units in a simple form.

\section{Conclusions}

In the given literature review, the description of phasor measurement units along with its optimal placement and potential application is discussed. It also discusses about how PMU will be detecting voltage instability with the use of various techniques. Based upon the application, any one of these technique can be chosen. Here the brief idea of several techniques of PMU placement is given. The objective of all the techniques remains same i.e.; to have optimal number of PMUs placement which will minimize the cost as well as necessity of PMU at all the buses.

\section{References}

1. Singh, et. al. International Journal of Engineering, Sciences and Technology. 2011; 3(3):64-82.

2. Jaime De La Ree, Virgilio Centeno, James S. Thorp, Phadke AG. Synchronized Phasor Measurement Applications in Power Systems. IEEE Transactions on Smart Grid. 2010 Jun; 1(1).

3. Jason Sexauer, Pirooz Javanbakht, Salman Mohagheghi. IEEE, Phasor Measurement Units for the Distribution Grid: Necessity and Benefits, 2013.

4. Phadke AG. Synchronized Phasor measurements. IEEE Computer Applications in Power, 1993 Apr.

5. Chen J, Abur A. Placement of PMUs to enable bad data detection in state estimation. IEEE Trans. Power Systems. 2006 Nov; 21(4):1608-15.

6. $\mathrm{Xu} \mathrm{B}$, Yoon YJ, Abur A. Optimal placement and utilization of phasor measurements for state estimation, PSERC Publication, 2005 Oct, p. 05-20.

7. Gou B. Generalized integer linear programming formulation for optimal PMU placement. IEEE Trans. Power Systems. 2008 Aug; 23(3):1099-1104.

8. Sodhi R, Srivastava SC. Optimal PMU placement to ensure observability of power system. 15th National Power Systems Conference (NPSC), IIT Bombay, 2008 Dec.

9. Chakrabarti S, Kyriakides E, Eliades DG. Placement of synchronized measurements for power system observability. IEEE Trans. Power Delivery. 2009 Jan; 24(1):12-19.

10. Chakrabarti S, Kyriakides E, Albu M. Uncertainty in power system state variables obtained through synchronized measurements. IEEE Trans. Instrumentation and Measurement. 2009 Jan; 58(8):2452-58.

11. Zhou M. Centeno VA, Phadke AG, Hu Y, Novosel D, Volskis HAR. A preprocessing method for effective PMU placement studies. $3^{\text {rd }}$ IEEE Int. Conf. on Electric Utility Deregulation and Restructuring and Power Technologies, 2008 Apr, p. 2862-67.

12. Yang Y, Shu H, Yue L. Engineering practical method for PMU placement of 2010 Yunnan power grid in China. In: 2009 Int. Conf. On Sustainable Power Generation and Supply, p. 1-6.

13. Antonio AB, Torreao JRA, Do Coutto Filho MB. Meter placement for power system state estimation using simulated annealing. In: Proc 2001 IEEE Power Tech. 
14. Kerdchuen T, Ongsakul W. Optimal PMU placement by stochastic simulated annealing for power system state estimation. GMSARN International Journal. 2008 Jun; 2(2):61-66.

15. Kerdchuen T, Ongsakul W. Optimal PMU placement for reliable power system state estimation. 2nd GMSARN Int. Conf., Pattaya, Thailand, 2007.

16. Gamm AZ, Kolosok IN, Glazunova AM, Korkina ES. PMU placement criteria for EPS state estimation. In: 2008 Int. Conf. on Electric Utility Deregulation and Restructuring and Power Technologies, p. 645-49.

17. Gavrilas M, Rusu I, Gavrilas G, Ivanov O. Synchronized phasor measurements for state estimation. Revue Roumaine des Sciences Techniques. 2009; 4:335-44.

18. Marín FJ, García-Lagos F, Joya G, Sandoval F. Optimal phasor measurement unit placement using genetic algorithms. Computational Methods in Neural Modeling. 2003; 2686:486-93.

19. Peng J, Sun Y, Wang HF. Optimal PMU placement for full network observability using Tabu search algorithm. International Journal of Electrical Power and Energy Systems. 2006 May; 28(4):223-31.

20. Aminifar F, Lucas C, Khodaei A, Fotuhi Firuzabad M. Optimal placement of phasor measurement units using immunity genetic algorithm. IEEE Trans. Power Delivery. 2009 Jul; 24(3):1014-20.

21. Gao Y, Hu Z, He X, Liu D. Optimal placement of PMUs in power systems based on improved PSO algorithm. In: 2008 IEEE IntConf. on Industrial Electronics and Applications, p. 2464-69.

22. Hajian M, Ranjbar AM, Amraee T, Mozafari B. Optimal placement of PMUs to maintain network observability using a modified BPSO algorithm. International Journal Electrical Power and Energy Systems. 2011 Jan; 33(1):28-34.

23. Peng $\mathrm{C}, \mathrm{Xu} \mathrm{X}$. A hybrid algorithm based on BPSO and immune mechanism for PMU optimization placement. In: 2008 World Cong. On Intelligent Control and Automation, p.7036-40.
24. Bo W, Discen L, Li X. An improved ant colony system in optimizing power system PMU placement problem. In: 2009 Asia- Pacific Conf. on Power and Energy Engineering, p. 1-3.

25. Kundur P, et. al. Definition and classification of power system stability. IEEE Transactions on Power Systems. 2004 May; 19(2):1387-1401.

26. Sudha R, Deepak Jain, Umang Lahoty, Swati Khushalani, Nivedita G, Jayabarathi T. State Estimation and Voltage Stability Monitoring Using ILP PMU Placement. International Journal of Soft Computing and Engineering (IJSCE). 2012 May; 2(2). ISSN: 2231-2307.

27. Heng-Yi Su, Chih-Wen Liu. Estimating the Voltage Stability Margin Using PMU Measurements. IEEE Transactions on Power Systems.

28. Xiaoming Mou, Weixing Li, Zhimin Li. PMU Placement for Voltage Stability Assessment and Monitoring of Power Systems. 2012 IEEE 7th International Power Electronics and Motion Control Conference - ECCE Asia June 2-5, 2012, Harbin, China.

29. Worawat Nakawiro, István Erlich. On-line Voltage Stability Load Index Estimation Based on PMU Measurements. DRPT2008 6-9, 2008 Apr, Nanjing China

30. Kesherwani S, Satyendra P. Singh, Singh SP. Voltage Stability Assessment using Phasor Measurement Units in Power Network with Full System Observability. 2nd International Conference on Power, Control and Embedded Systems, 2012.

31. LIU Chengxi, CHEN Zhe, Claus Leth Bak, LIU Zhou. Adaptive Voltage Stability Protection Based on Load Identification Using Phasor Measurement Units. The International Conference on Advanced Power System Automation and Protection, 2011.

32. Gupta BR. Power system analysis and design. Fourth edition, 2006, p 209-269. ISBN: 81-219-2238-0.

33. Phadke AG, Thorp JS. Synchronized Phasor Measurements and Their Applications. Digital Control of Electrical Drives. ISBN 978-0-387-76535-8 